\title{
Developmental neuroscience: an important step for understanding child and adolescent mental disorders
}

\author{
Beate Herpertz-Dahlmann
}

Published online: 9 August 2012

(C) Springer-Verlag 2012

The prevalence rates of child and adolescent mental health problems range from 10 to $20 \%$ worldwide (German Health Interview and Survey, Ravens-Sieberer et al. 2008; Srinath et al. 2010, NIMH 2001), implying an enormous personal and societal toll. The increasing public interest in these disorders (e.g., NIMH 2001) underlines the need for insight into the origin of these disorders and the development of effective therapeutic and preventive strategies. During the last two decades, accelerated biological research in child and adolescent psychiatry has contributed to a better understanding of the mechanisms that underlie the occurrence of mental illness. Progress has been made in genetics and epigenetics, neuropsychology, neuroendocrinology, neuroimaging, and other areas. Several examples from developmental neuroscience are provided in this issue to elucidate this progress and to describe multifactorial models of the emergence of psychiatric disorders in youth.

Three articles in this issue offer different approaches to advance the understanding of risk factors that contribute to the development of psychopathology in childhood and adolescence (Legenbauer et al., Ise et al., Dahmen et al.).

In the study by Legenbauer et al., a recently defined syndrome called "severe mood dysregulation" and its association with sleep disturbances is investigated. This phenotype is characterised by severe, protracted irritability and hyperarousal that is normally symptomatic of mania but that does not fulfil other characteristics of bipolar disorder, especially clearly demarcated episodes of mood changes (Leibenluft 2011). In the past-in addition to

B. Herpertz-Dahlmann ( $\square)$

Department of Child and Adolescent Psychiatry, Psychosomatics and Psychotherapy, RWTH Aachen University,

Neuenhofer Weg 21, 52074 Aachen, Germany

e-mail: bherpertz-dahlmann@ukaachen.de bipolar disorder-children with this syndrome were often diagnosed as having ADHD. However, recent research has elucidated significant differences in psychopathology, pathophysiology, family history and outcome. Nevertheless, sleep disorders are also often a symptom of ADHD and bipolar disorder. The authors investigated a large epidemiological sample of more than 4,500 9-year-old school children and demonstrated that children suspected of this newly defined syndrome had significantly more sleep disturbances than their healthy counterparts. Although the type of sleep disorder in this study did not differ from that of ADHD or bipolar disorder and thus may not be validated as a "biological marker", sleep problems must be addressed in children seeking help for this disabling disorder.

In the paper by Ise et al., spelling disorder, a neurocognitive impairment, is the focus, with a concentration on neuropsychological risk factors. In line with other learning disorders (without restriction of general intelligence), such as reading and mathematics disorders, the present study emphasises a multifactorial view of this disturbance. This disorder often has a severe impact on a child's development, including low educational achievement and emotional and social problems. The authors show that children with poor spelling performance have difficulties in implicit learning, suggesting that these learning processes are important for the acquisition of general literacy skills. Considering the subtypes of learning impairments, progress in treatment of these disorders will also depend on identifying cognitive profiles to "personalise" treatment strategies.

In the last article on risk factors (Dahmen et al.), the authors present recent knowledge to explain how adverse environment (in this case, lack or loss of care) influences brain maturation. Children with so-called reactive attachment 
disorder often display ADHD-like features. This may be due to overactivity of the stress system under "pathogenic care", resulting in dysfunction of the prefrontal cortex and the hippocampus. In their review, these authors describe how "nature" interacts with "nurture"-that is, how neglect and/or abuse affect hormonal and other neurochemical pathways in brain development as well as subsequent behaviour.

Eating disorders, particularly anorexia nervosa, have morbidity and mortality rates that are the highest of any mental disorders. In contrast to the "psychosomatic family model" of 20 years ago, anorexia nervosa is now seen as a complex neuropsychiatric disorder with an important genetic component that is likely expressed primarily in childhood by specific traits, such as inhibition, perfectionism and harm avoidance (Herpertz-Dahlmann et al. 2011). The eating disorder is an excellent example of the interaction of genetics and disorder-induced long-lasting sequelae; in other words, starvation itself is associated with severe neuroendocrine and neurotransmitter changes that affect the brain. In the present study (Bühren et al.), the authors investigate set-shifting abilities in adolescent patients with anorexia nervosa, which are closely related to rigidity and obsessive-compulsive traits. They compare these abilities with those of adult patients to clarify the influence of chronicity and brain maturation.

One article released in this issue refers to more basic science (Dingerkus et al.). The serotonergic neurotransmitter system plays an important role in regulating elementary functions, such as appetite and sleep, but it also plays a role in the pathogenesis of several psychiatric disorders. Acute tryptophan depletion (ATD) by administering an amino acid beverage without tryptophan is an effective and short-acting method to affect serotonergic functions in the brain. In their investigation, the authors verify that their method of weight-adapted tryptophan depletion (Moja-De), which can also be used in children and adolescents, significantly reduces tryptophan influx into the CNS. This may prove to be an important and safe tool to modulate serotonin metabolism and to gain further insight into serotonin-associated mood states and disorders.

How well do treatments work for young patients? Fortunately, this issue also includes a treatment study. Autism is a pervasive developmental disorder that has increasingly been diagnosed in children. It is characterised by a restricted repetitive and stereotyped pattern of behaviour, communication deficits and impaired social interaction. Autism was long thought to be treatment resistant. However, in recent years, various intervention programmes have been developed. Because of the enormous plasticity of the young human brain, these programmes seem to be especially helpful during the toddler and preschool years. The present paper introduces the first pre-post comparison of a developmentally based structured behavioural training programme for autistic children in combination with training of parents and kindergarten teachers. The intervention resulted in an improvement in social skills and behaviour as well as an increase in the mental age quotient/IQ, although the gains were smaller in comparison to the classic "Applied Behaviour Analysis (ABA)" therapy. However, the latter is much more demanding for both the child and the parents and is highly cost and time intensive. In the near future, significant efforts must be made to develop more effective intervention programmes for this increasingly prevalent and debilitating disorder.

Together, these papers improve understanding of the neurobiological and neurocognitive mechanisms by which child and adolescent mental illness emerges and how it can be treated. Understanding these mechanisms may facilitate the development of proactive prevention and the establishment of early intervention strategies rather than treatment in response to symptoms later in life. Thus, in the near future, hopefully, developmental neuroscience may further accelerate steps to overcome the burden of mental illness in youth.

\section{References}

Herpertz-Dahlmann B, Seitz J, Konrad K (2011) Aetiology of anorexia nervosa: from a "psychosomatic family model" to a neuropsychiatric disorder? Eur Arch Psychiatry Clin Neurosci 261:177-181

Leibenluft E (2011) Severe mood dysregulation, irritability, and the diagnostic boundaries of bipolar disorder in youths. Am J Psychiatry 168:129-142

National Institute of Mental Health (2001) Blueprint for change. Research on child and adolescent mental health. Washington, USA

Ravens-Sieberer U, Wille N, Erhart M, Bettge S, Wittchen HU, Rothenberger A, Herpertz-Dahlmann B, Resch F, Hölling H, Bullinger M, Barkmann C, Schulte-Markwort M, Döpfner M, BELLA study group (2008) Prevalence of mental health problems among children and adolescents in Germany: results of the BELLA study within the National Health Interview and Examination Survey. Eur Child Adolesc Psychiatry 17:22-33

Srinath S, Kandasamy P, Golhar TS (2010) Epidemiology of child and adolescent mental health disorders in Asia. Curr Opin Psychiatry 23:330-336 